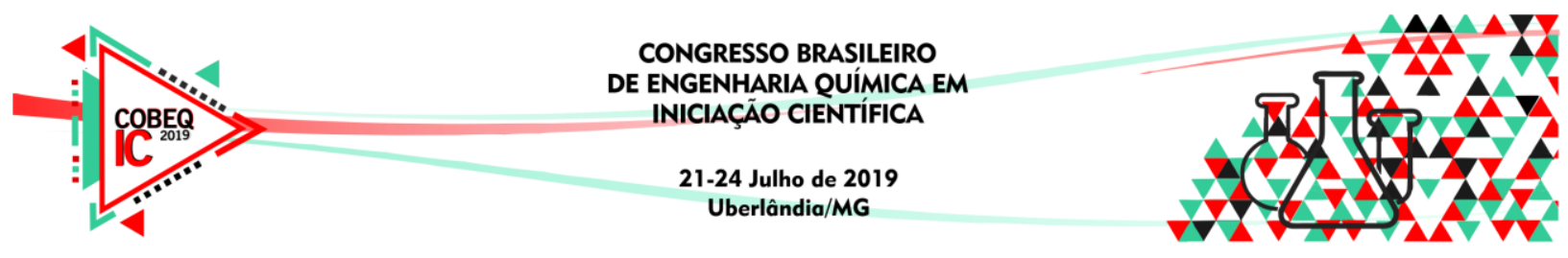

\title{
ANÁlise DO PRECOATING NA FILTRAÇÃo DE PÓ DA ACIARIA DE UMA INDÚSTRIA SIDERÚRGICA
}

\author{
R. P. M. A. ARANHA ${ }^{1}$, R. SARTIM ${ }^{2}$, M. L. AGUIAR ${ }^{1}$, \\ ${ }^{1}$ Universidade Federal de São Carlos, Departamento de Engenharia Química \\ ${ }^{2}$ Universidade Federal do Espírito Santo \\ E-mail para contato: renan.marq.aranha@ @otmail.com
}

\begin{abstract}
RESUMO - Um dos equipamentos muito utilizado nas indústrias para controlar a emissão de poluentes no ar é o filtro de mangas. Esse equipamento possui elevada eficiência para as partículas micrométricas e aplicação em diferentes tipos de processos, além de possuir baixo custo de operação. Para aumentar a eficiência de coleta de partículas submicrométricas (200 a $300 \mathrm{~nm})$, algumas indústrias utilizam o pré-revestimento ou precoating, que visa preencher os poros do meio filtrante com partículas, e dessa forma melhorar o desempenho da filtração e prolongar a vida-útil da manga. Assim, o presente trabalho propôs estudar o efeito do precoating na eficiência de filtração, durante a operação de filtração. Para tal finalidade foram utilizados diferentes pós para os precoatings e o material particulado utilizado na operação de filtração foi proveniente de um determinado processo de uma indústria siderúrgica. Todos os materiais particulados utilizados os do precoats e o pó da siderúrgica foram caracterizados em relação à massa específica e o diâmetro médio volumétrico. Os ensaios, para avaliação da emissão de particulados, foram realizados utilizando um aparato experimental baseado na norma VDI 3926. Portanto, os ensaios mostraram que os meios filtrantes com o precoating obtiveram eficiência superior à membrana PTFE para partículas inferiores à $3 \mu \mathrm{m}$. Sendo o talco, o precoat, com o melhor desempenho.
\end{abstract}

\section{INTRODUÇÃO}

Devido ao aumento da poluição do ar, que dependendo das condições climáticas podem ser ainda maiores, dos meios de fiscalização, com leis mais rígidas, e da pressão da população, as indústrias estão mudando seu comportamento buscando equipamentos mais eficientes na separação gás-sólido. Entre os equipamentos mais utilizados para alcançar tais exigências, de acordo com Cirquiera (2017), os filtros de tecido (filtro de mangas) vem ganhando destaque, devido a sua alta eficiência de remoção de partículas finas e ultrafinas, chegando a alcançar eficiências superiores a 99\% dependendo do tamanho da partícula.

Com a pressão para reduzir as emissões de materiais particulados, os fabricantes de meios filtrantes desenvolverem novos filtros capazes de remover as partículas finas. Atualmente, o meio filtrante com as suas fibras recobertas por uma fina camada de membrana de politetrafluoretileno (PTFE), conhecido comercialmente como Teflon ${ }^{\circledR}$, é um dos mais utilizados quando se pretende obter alta eficiência de coleta para material particulado fino (acima de 99,99\%). Testes realizados por Barnett (2000) mostraram uma grande diminuição nas emissões de pó e na perda de carga utilizando meios filtrantes com a membrana. 


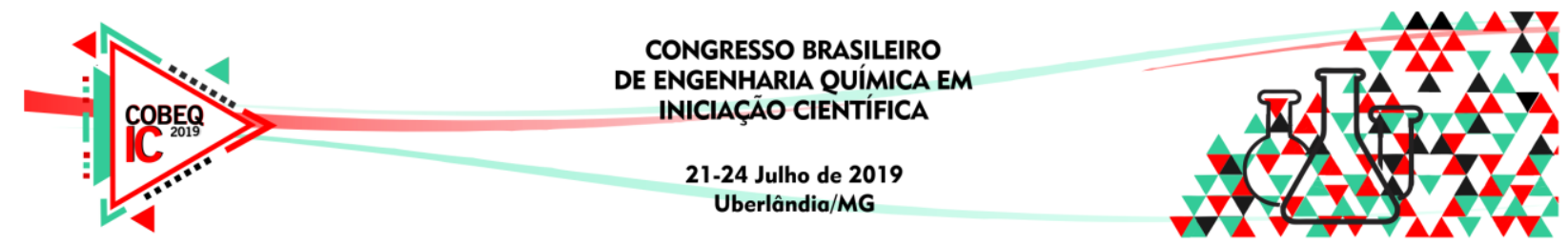

Entretanto, esses meios filtrantes apresentam custo relativamente alto, assim uma alternativa menos onerosa é o precoating.

O precoating, conforme Ravert (2005), consiste em recobrir o interior e a superfície de não-tecidos com material particulado formando uma camada de pó inicial a qual previne as partículas de penetrarem o meio filtrante e causar o entupimento. Além de garantir o escoamento do ar livremente pela manga, melhora a performance das mangas, aumenta sua vida útil e facilita a limpeza, promovendo melhor desprendimento da torta do meio filtrante.

No entanto, existem poucos estudos que relatam a caracterização de pó utilizado, como: o diâmetro, a quantidade de massa depositada no meio filtrante, a velocidade de escoamento e o tempo necessário para que o método seja mais eficiente. Pois é importante que as partículas do pó de precoating possuam tamanho adequado para não causarem o entupimento da manga, além de garantir baixa queda de pressão, alta permeabilidade e eficiência de coleta.

Dessa forma, o presente trabalho tem por objetivo realizar uma análise da eficiência do precoating na filtração de gases, utilizando um equipamento experimental baseado na norma VDI 3926. Para tal finalidade, utilizou-se como material particulado do precoating: o Talco, a Cal Virgem e o Calcário Dolomítico e para filtração o pó de aciaria de uma indústria siderúrgica.

\section{MATERIAIS E MÉTODOS}

\subsection{Caracterização dos Materiais Particulados}

Os materiais particulados utilizados nesse estudo foram: Pó de Aciaria, usado na filtração de gases, e o Talco, a Cal Virgem e o Calcário Dolomítico como pó do precoating. Para todos os pós realizou-se a análise de massa específica, em triplicata, pelo método de picnometria a hélio no equipamento AccuPyc 1330 Micrometrics. Os valores dos diâmetros mediano volumétrico foram determinados pelo equipamento Malvern Mastersizer Microplus.

\subsection{Caracterização dos Meios Filtrantes}

Como meio filtrante empregou-se os tecidos de poliéster com gramatura de $550 \mathrm{~g} / \mathrm{cm}^{2}$ (PE 550) e de poliéster com membrana de PTFE de $550 \mathrm{~g} / \mathrm{cm}^{2}$. Para determinar as médias dos diâmetros das fibras de cada amostra analisada, foram geradas imagens da superfície dos meios filtrantes utilizando um Microscópio Eletrônico de Varredura (MEV), do Laboratório de Caracterização Estrutural (LCE). A partir destas imagens, utilizou-se o software Image Pro Plus 7.0, para analisar as imagens, e posteriormente determinar os diâmetros das fibras. (Ergun):

A porosidade também foi estimada, para cada meio filtrante, a partir da Equação 1

$$
\frac{\Delta P}{L_{T}}=150 \cdot \frac{(1-\varepsilon)^{2}}{\varepsilon^{3}} \cdot \frac{\mu V_{f}}{d_{p}{ }^{2}}+1,75 \cdot \frac{(1-\varepsilon)}{\varepsilon^{3}} \cdot \frac{\rho_{g} V_{f}^{2}}{d_{p}}
$$




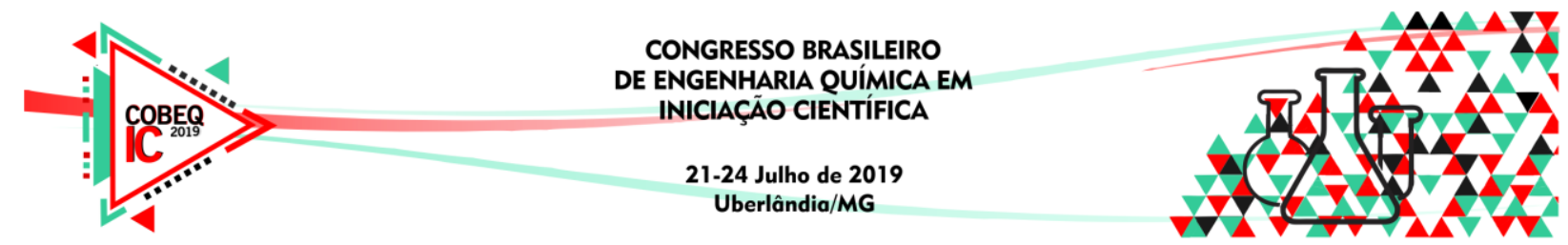

Para determinar a eficiência do meio filtrante para partículas menores que 10 micras, utilizou-se o aparato experimental apresentado na Figura 1, composto por um gerador de aerossol modelo 3433, um diluidor de aerossol 3302 e um espectrômetro Aerodynamic Particle Sizer (APS) modelo 3320 da TSI e uma bomba de vácuo.

Inicialmente o material particulado depositado em um prato rotativo do alimentador de partículas juntamente com o ar comprimido era arrastado com o auxílio de uma bomba de sucção para o sistema de filtração (meio filtrante e rotâmetro). Foram realizadas amostragens isocinéticas antes e após o meio filtrante. Posteriormente, o ar sujo passava por um diluidor, que continha um filtro HEPA para filtrar o ar ambiente succionado, e logo em sequência por um contador de partículas, no qual caracterizava as amostras em função do diâmetro aerodinâmico. $\mathrm{O}$ ar que escoava no diluidor foi calibrado, através de um rotâmetro até que se atingisse a vazão desejada. De acordo com Carvalho (2018) os meios filtrantes possuíam uma área útil de filtração de $17,57 \mathrm{~cm}^{2}$, e a relação ar-pano empregada foi de $2 \mathrm{~m} / \mathrm{min}$.

Figura 1: Aparato experimental para avaliação da emissão de particulados.

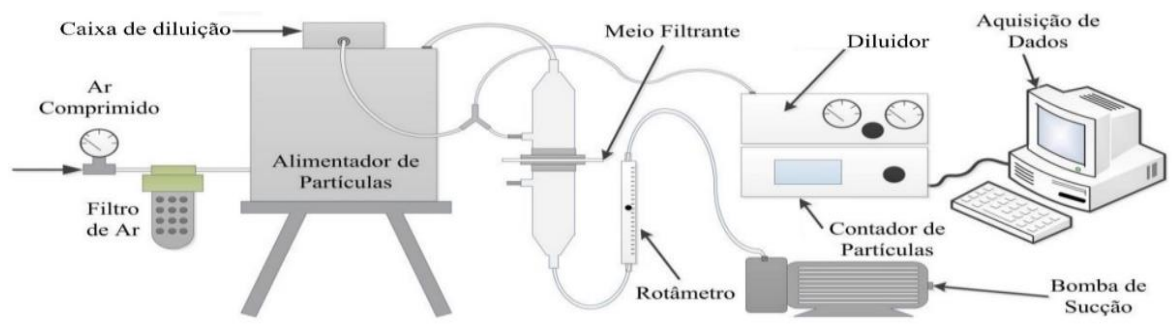

Fonte: Adaptado de Cirqueira (2017)

O trabalho de Carvalho (2018) mostra que os ensaios consistiram em coletar amostras do pó da aciaria antes e depois do meio filtrante ao longo da filtração, conforme a queda de pressão no filtro submetido ao precoating: $\Delta \mathrm{P}_{0}+10 \mathrm{mmH}_{2} \mathrm{O}, \Delta \mathrm{P}_{0}+20 \mathrm{mmH}_{2} \mathrm{O}$ e $\Delta \mathrm{P}_{0}+30$ $\mathrm{mmH}_{2} \mathrm{O}$. A partir da análise de eficiência de coleta, obtida pela Equação 2 para as diferentes faixas de diâmetro aerodinâmico, determinou-se a queda de pressão capaz de alcançar uma eficiência de coleta próxima a 100\% para partículas submicrométricas (200 a $300 \mathrm{~nm}$ ).

$E=\frac{C_{i}-C_{f}}{C_{i}} \cdot 100 \%$

\section{RESULTADOS E DISCUSSÃO}

Os valores de massa específica e do diâmetro mediano volumétrico dos materiais particulados utilizados no precoating e na filtração estão apresentados na Tabela 1 e foram obtidos por Andrade (2019). Os valores do pó de aciaria utilizado na filtração, após o precoating, foi obtido por Carvalho (2018). 


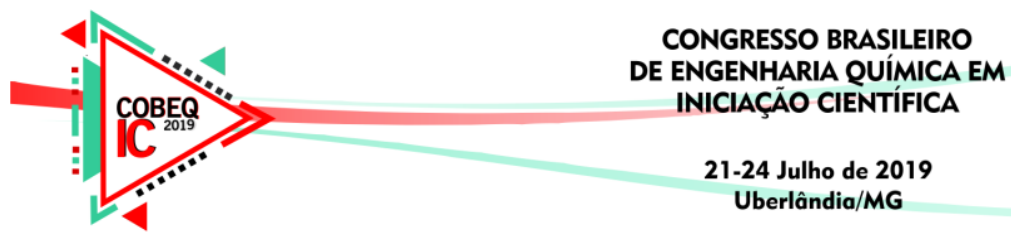

Tabela 1 - Distribuição volumétrica e Massa específica dos materiais particulados

\begin{tabular}{|c|c|c|c|c|}
\hline Distribuição & Cal hidratada & Calcário Dolomítico & Talco & Pó de aciaria \\
\hline $\mathrm{D}(\mathrm{v}, 0,1) \mu \mathrm{m}$ & $0,423 \pm 0,006$ & $0,49 \pm 0,04$ & $6,52 \pm 0,02$ & $0,38 \pm 0,01$ \\
\hline $\mathrm{D}(\mathrm{v}, 0,5) \mu \mathrm{m}$ & $22,18 \pm 0,87$ & $27,9 \pm 0,81$ & $25,09 \pm 0,09$ & $19,5 \pm 0,13$ \\
\hline $\mathrm{D}(\mathrm{v}, 0,9) \mu \mathrm{m}$ & $99,15 \pm 9,93$ & $128,27 \pm 5,16$ & $56,72 \pm 0,55$ & $117,52 \pm 18,93$ \\
\hline$\rho\left(\mathrm{g} / \mathrm{cm}^{3}\right)$ & $2,7742 \pm 0,0014$ & $2,8422 \pm 0,0010$ & $3,0061 \pm 0,0012$ & $3,4179 \pm 0,0043$ \\
\hline
\end{tabular}

Observa-se na Tabela 1 que tanto os pós do precoating como o pó da aciaria apresentam uma distribuição bastante polidispersa. O pó da aciaria apresenta um diâmetro mediano menor $(19,5 \mu \mathrm{m})$ que os pós utilizados no precoating (de 22 a $28 \mu \mathrm{m})$.

Os dados referentes aos diâmetros médios das fibras e da porosidade dos meios filtrantes estão apresentados na Tabela 2 e foram obtidos por Andrade (2019).

Tabela 2 - Diâmetros médios e Porosidade dos meios filtrantes

\begin{tabular}{|c|c|c|}
\hline Meio Filtrante & Diâmetro médio das fibras $(\mu \mathrm{m})$ & Porosidade \\
\hline PE $550 \mathrm{~g} / \mathrm{cm}^{2}$ & $14,9 \pm 3,9$ & $0,883 \pm 0,0026$ \\
\hline PE + PTFE 550 g/cm ${ }^{2}$ & $5,10 \pm 5,7$ & $0,999 \pm 0,013$ \\
\hline
\end{tabular}

Observa-se na Tabela 2 que a porosidade do meio filtrante de poliéster com membrana de PTFE foi maior que os de poliéster sem a membrana. Isto já era esperado, uma vez que as fibras com membrana de PTFE apresentam diâmetros menores que as de poliéster.

As eficiências de coleta em função do diâmetro aerodinâmico para micropartículas são apresentadas na Figura 2. Comparando o meio filtrante com a membrana de PTFE com o de poliéster de mesma gramatura, verifica-se que a membrana foi responsável por um aumento significativo na eficiência de coleta para partículas com diâmetro inferior a $3 \mu \mathrm{m}$, mesmo apresentando uma porosidade maior. Esse comportamento também foi verificado no trabalho Bortolassi, Guerra \& Aguiar (2015), que observaram que as partículas tenderam a se depositar mais facilmente nas fibras de menores diâmetros e Podgórski, Balazy \& Gradón (2006) avaliaram um meio filtrante convencional e cinco filtros com nanofibras e os resultados dos experimentos confirmaram que meios filtrantes contendo nanofibras possuem maiores eficiências de filtração.

Figura 2- Eficiência de coleta dos meios filtrantes para o pó da aciaria

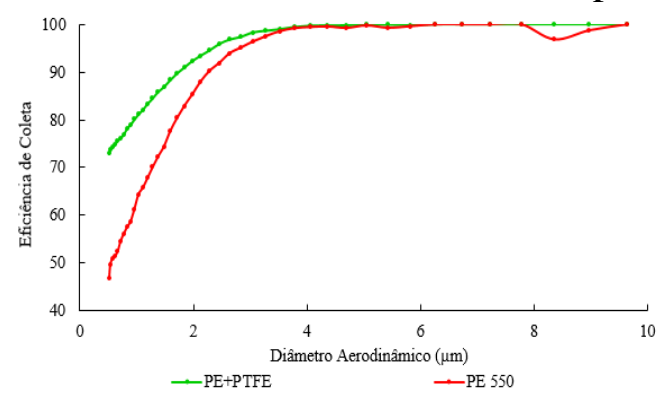

As Figuras 3, 4 e 5 ilustram as eficiências de coleta para o meio filtrante de poliéster após o precoating com a cal hidratada, o calcário dolomítco e o talco, respectivamente, 


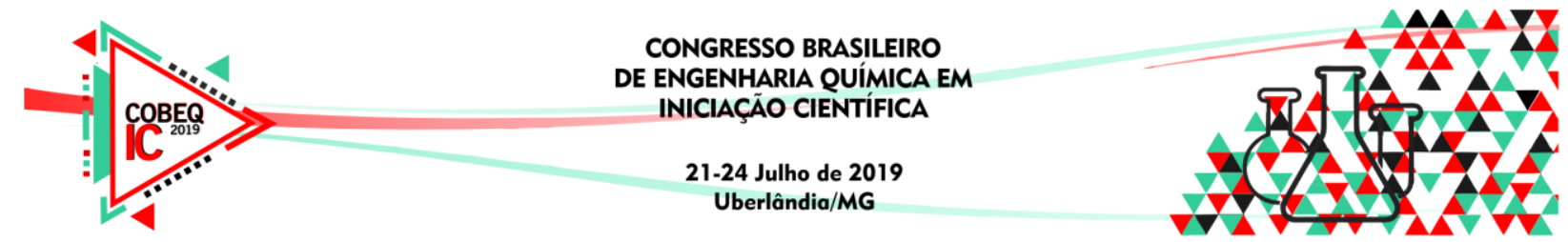

variando a perda de carga limite do processo de precoating. Observa-se na Figura 3, com o pó de precoating de cal hidratado, que a eficiência ficou próxima de $100 \%$, para partículas acima de $4 \mu \mathrm{m}$ para todas as camadas de precoating aplicadas, sendo que para partículas abaixo de 3 $\mu \mathrm{m}$ a eficiência para camada de $10 \mathrm{mmH}_{2} \mathrm{O}$ foi diminuindo até chegar em $85 \%$. Já para o precoating com o calcário dolomítico (Figura 4) a eficiência ficou acima de $96 \%$ para todas as camadas de precoating, para os diâmetros variando de 0 a $20 \mu \mathrm{m}$, sendo que para a camada de $10 \mathrm{mmH}_{2} \mathrm{O}$ teve o pior desempenho para partículas menores que $3 \mu \mathrm{m}$. Entretanto, para o precoating de talco, a eficiência de coleta ficou muito próxima a $100 \%$ independente da camada de talco aplicada na superfície do meio filtrante, apresentando a melhor performance. Portanto, dependendo do material particulado utilizado no precoating não é necessário aplicar camadas mais espessas desse pó, uma fina camada de precoating já é o suficiente para aumentar a eficiência de coleta para partículas superfinas. Verifica-se também que a composição química e física da partícula deve ser analisada antes de se escolher o pó do precoating a ser utilizado na filtração de filtros de mangas.

Figura 3 - Eficiência de coleta variando a perda de carga limite do precoating para cal hidratado.

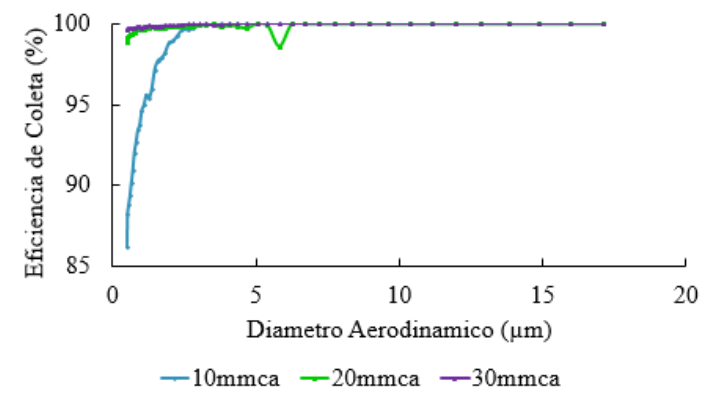

Figura 4 - Eficiência de coleta variando a perda de carga limite do precoating para calcário dolomítico.

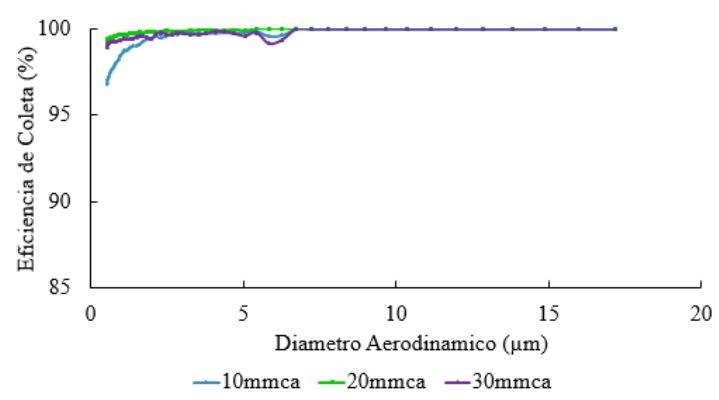

Figura 5 - Eficiência de coleta variando a perda de carga limite do precoating para talco.

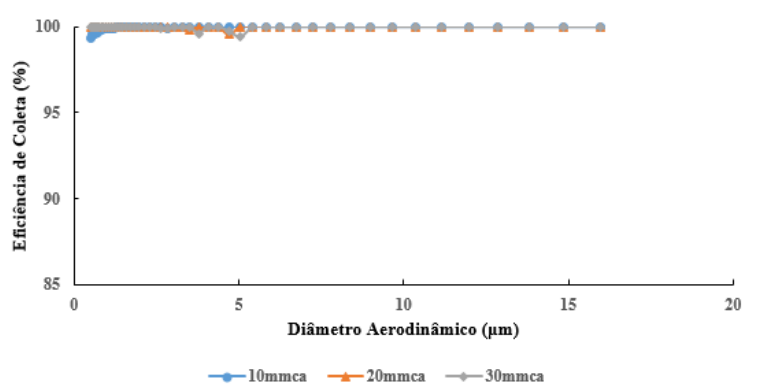




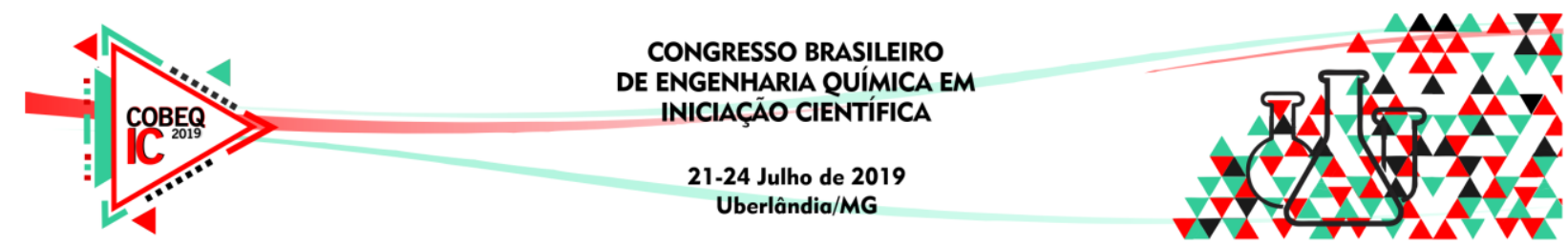

\section{CONCLUSÕES}

A porosidade do meio filtrante de poliéster com membrana de PTFE foi maior que o de poliéster sem a membrana, mesmo os filtros apresentando a mesma gramatura.

Para a eficiência de coleta de micropartículas, o meio filtrante com membrana de PTFE, com maior porosidade mostrou-se mais eficiente para partículas menores do que $3 \mu \mathrm{m}$. Dessa forma o diâmetro da fibra teve maior influência na eficiência de coleta, sendo a fibra de menor diâmetro a mais eficiente.

Em relação ao precoating, os testes de perda de carga máxima indicaram alta eficiência de coleta, acima de $98 \%$, a partir da perda de carga máxima de $20 \mathrm{mmH}_{2} \mathrm{O}$, tanto para a cal hidratada quanto para o calcário dolomítico e o talco. Sendo que para o talco o desempenho foi melhor independente da camada aplicada

Os meios filtrantes com o precoating obtiveram eficiência superior à membrana PTFE para partículas inferiores à $3 \mu \mathrm{m}$, mostrando ser uma ótima alternativa e mais econômica para aumentar a eficiência de filtração dos filtros de mangas.

\section{NOMENCLATURAS}

D - Distribuição

$$
\rho \text { - Massa específica }\left[\mathrm{M} \mathrm{L}^{-3}\right] \quad \Delta \mathrm{P}_{0} \text { - Perda de carga inicial }
$$

\section{REFERÊNCIAS}

ANDRADE, B. K. S. A., Avaliação do precoating e dos meios filtrantes para filtro de mangas do processo de despoeiramento secundário da aciaria, , São Carlos, 2019.

BARNETT, T. "Improving the performance of fabric filter dry dust collection equipment" Filtration and Separion, pp. 28 - 32, 2000.

BORTOLASSI, A. C. C.; GUERRA, V. G.; AGUIAR, M. L., Eficiência e permeabilidade de diferentes meios filtrantes na remoção de nanopartículas através da variação do tempo em soluções de NaCl e de sacarose, Fev. 2015 vol. 1 num. 2 - XX COBEQ.

CARVAlHO, M. V. A., Avaliação de meios filtrantes para filtro de mangas com Sistema de limpeza de jato de ar pulsante do processo de despoeiramento secundário da aciaria de uma indústria siderúrgica, São Carlos, 2018.

CIRQueIRA, S. S. R.; TANABE, E. H.; AGUIAR, M. L. Process Safety and Environmental Protection, v. 105, p. 69-78, jan. 2017.

PODGÓRSKY, A., BALAZY, A., GRADÓN, L. Aplication of nanofibers to improve the filtration efficiency of the most penetrating aerossol particles in fibrous filters. Published in Chemical Engineering Science - v. 61, pp. 6804-6815.

RAVERT, E. Precoating new filters for better airflow, longer filter life. UAS 